\title{
Local esperience on frequency and significance of immunological overlap in the field of primary biliary cirrhosis (PBC)
}

\author{
Caterina Defendenti', Simona Bollani², Gabriella Munegato', Simone Saibeni², \\ Maria Sofia ${ }^{3}$, Savino Bruno ${ }^{2}$, Mariella Saudelli', Maurizio Picca ${ }^{3}$ \\ 'Laboratorio analisi, Ospedale Fatebenefratelli, Milano \\ ${ }^{2}$ Ambulatorio di Epatologia, Ospedale Fatebenefratelli, Milano \\ ${ }^{3}$ Unità di Medicina Interna, Ospedale Macedonio Melloni, Milano
}

Local esperience on the frequency and significance of immunological overlap in the field of primary biliary cirrhosis (PBC)

Key words: Overlap syndromes, Primary biliary cirrhosis, Serum autoantibodies, Autoimmunity

\section{SUMMARY}

The aim of this study was to investigate the frequency and quality of immunological overlap in serum AMA M2 positives. During the period January 2004 - December 2006, out of 3000 sera evaluated, 40 were AMA M2 positive. Six of these (15\%) were without immunological overlap; they had clinical, histological and biochemical features of classical PBC with a normal length of life and ursodeoxycholic acid responding. In other 16 patients (40\%), there was an association with antibody against antigens as SLA/LP, gp2 10 and Sp I00, that causes the overlap syndrome AlH/PBC with various level of seriousness. The association with anti SLA/LP or with high level of autoantibody anti-Sp 100 (index ELISA>10) waspresent in the patients investigated, as AlH type $\mathrm{I}^{\circ}$. Patients positive for anti gp2 10 with low anti-Sp 100 index had instead a weak overlap AlH/PBC with slow evolving aspects. Nobody was positive for anti-LKMI, anti-LC, PML, or LBR. The remaining $45 \%$ of sera were positive for ANA, ENA,ANCA, with different clinical features.

The antibody anti-SSARo52 and anti-centromere were frequent (17\%) and modified the liver disease evolution. The most serious clinical case was a double overlap anti-centromere/SSARo52 with biochemical and histological AlH symptoms. Other ENA patterns did worsen the prognosis of PBC but showed or were associated with systemic diseases, often after a long asymptomatic period.The ANA IFI positive with a speckled pattern ENA negative, in other 7 patients (17\%) worsened the long term prognosis because they expressed an overlap AlH/PBC with slowly evolving aspects. The most serious and intense clinical picture, with transplant perspective, was the association of the AMA M2 with anti SpI00 (index > 10) and pANNA. Four case were male (10\%) but females were prevalent.

\section{INTRODUZIONE}

La presenza di autoanticorpi antimitocondrio (AMA) con specificità anti M2 si ha nel 90/95\% dei casi di cirrosi biliare primitiva (PBC), una malattia epatica colestatica, che clinicamente può rimanere silente anche molti anni dopo il rilievo della positività sierologia (11).

Dal punto di vista istopatologico la PBC è caratterizzata da infiammazione portale e distruzione immunomediata di dotti biliari intraepatici con vari gradi di severità e di rapidità di evoluzione. Rispetto ad altre malattie autoimmunitarie, si ha un attacco organo specifico nonostante l'antigene coinvolto sia presente in tutte le cellule nucleate (9).

Le alterazioni anatomiche si traducono in ridotta secrezione di bile e ritenzione di sostanze tossiche con danno epatico, fibrosi, cirrosi ed, eventualmente, insufficienza epatica.

Nelle forme non associate ad altre alterazioni immunologiche la malattia ha una evoluzione lentissima, è compatibile con una normale durata di vita, non richiede quindi necessariamente trattamento corticosteroideo ma ci si può limitare all'uso di acido urodesossicolico che aumenta il deflusso delle vie biliari e riduce l'evoluzione della flogosi cronica.

La presenza di AMA M2 è uno dei tre criteri su cui si basa la diagnosi di cirrosi biliare primitiva insieme al dato istologico (necessario anche per la stadiazione della malattia) e biochimico (elevati livelli di enzimi epatici per più di sei mesi).

In circa il 5-10\% di PBC non sono rilevabili anti- 
corpi anti mitocondrio pur essendo l'andamento della malattia esattamente sovrapponibile a quello dei pazienti sierologicamente positivi.

Nell'ultimo decennio un buon numero di strutture nucleari sono state riconosciute come target di ANA nella PBC. Alcune di esse danno quadri overlap che peggiorano la prognosi in quanto si associano ad una tendenza più aggressiva della malattia e necessitano di trattamento immunosoppressore. Il loro riconoscimento non ha quindi un'importanza trascurabile per la gestione di una malattia che può essere compatibile con una normale durata di vita. In alcuni casi la presenza di autoanticorpi verso patterns specifici può configurare un possibile overlap AIH/PBC.

$\mathrm{Nel}$ presente lavoro riportiamo l'esperienza di tre anni su un gruppo di pazienti, tutti AMA M2 positivi, di cui si vuole valutare la qualità e le percentuali di overlap immunologico con pattern ANA, ENA e ANCA e le eventuali ricadute sull'andamento clinico in base ai dati ematochimici disponibili.

Una ricerca il più possibile ampia delle specificità antigeniche coinvolte nella malattia autoimmune può, attualmente, essere di reale aiuto per il clinico anche alla luce di recenti sviluppi degli studi in materia.

\section{MATERIALI E METODI}

Popolazione allo studio

Sono stati analizzati parametri autoimmunitari su sieri di 40 pazienti risultati positivi per autoanticorpi anti AMA M2. I sieri, raccolti nell'arco di tre anni (2004-2006), appartengono a pazienti pervenuti al nostro laboratorio per controlli di routine, sono stati conservati alla temperatura di $-5^{\circ} \mathrm{C}$.

Per l'identificazione dei patterns immunologici sono stati utilizzati metodi diversi:

Screening ANA in immunofluorescenza indiretta: Per l'identificazione dei patterns classici nello screening routinario di laboratorio: ANA, AMA, ASMA, LKM, APGA sono stati utilizzati substrati HP2 e tre tessuti (fegato, rene, mucosa gastrica) combinati con quattro pozzetti su di un unico vetrino; diluizione 1/80 (Basic Profile, Euroimmun).

\section{ANCA in immunofluorescenza indiretta:}

Sono stati utilizzati vetrini di quattro pozzetti con adesi rispettivamente granulociti fissati in etanolo, granulociti fissati in formalina, tessuto epatico e cellule HP2; diluizione 1/80. (Granulocytes Mosaic; Euroimmun).

\section{ELIA MPO, PR3}

Per la determinazione delle due principali specificità anti neutrofili (MPO e PR3) è stato utilizzato un metodo immunoenzimatico con rilevazione in immunofluorescenza ELISA (Immunocap, Sweden).

ANCA-Combi

Test ELISA per la identificazione di ANCA atipici; riconosce elastasi, lattoferrina, lisozima, BPI, catepsina $\mathrm{G}$ (Chematil).

\section{ELISA Liver Profile S}

Test semiquantitativo con tecnica ELISA per la determinazione di LKM1, AMA M2, Sp100, LBR, SLA (Imtec-Liver Profile S)

\section{Immunblot liver}

Identificazione in contemporanea di antigeni correlati con epatopatie adesi a striscia di cellulosa:

Gp210, sp100, LC1, M2, LKM1, PML SSA-Ro52 (Immunoblot Liver, Euroimmun);

\section{Immunoblot ANA profile}

La valutazione qualitativa dei patterns ENA con metodo blott: nRNP/Sm, Sm, SS-A, SS-B, Scl70, PM-Scl, Jo-1, CENP B, dsDNA, Nucleosomi, istoni, proteina P-ribosomiale e AMA-M2 (Euroline ANA-Profile $3 \mathrm{IgG}$, Euroimmun).

\section{RISULTATI}

Su 3000 sieri con richiesta di autonticorpi anti AMA tra il 2004 e il 2006, n.40 (1.3\%) sono risultati positivi per AMA M2. L'età media era di 59 aa (35-87); il 90\% (n. 36) apparteneva a pazienti di sesso femminile ed il $10 \%$ (n. 4) a pazienti di sesso maschile.

$\mathrm{Si}$ è fatta una correlazione tra le specificità autoanticorpali riscontrate ed i livelli di interessamento epatico in base ai dati ematochimici disponibili nel nostro laboratorio. Per i pazienti in cura presso l'ambulatorio di epatologia si sono avute anche conferme di tipo clinico, istologico e strumentale.

Il 15\% dei campioni AMA M2 positivi non presentava alcun tipo di associazione con altri autoanticorpi, mentre nell' $85 \%$ dei casi è stato riscontrato overlap immunologico di cui si è voluto valutare la natura e la frequenza in rapporto alla rapidità evolutiva e alla gravità sintomatologica. La presenza di overlap con altre specificità autoanticorpali complica l'aspetto clinico e prognostico e va valutato con attenzione vista la necessità di diversi approcci terapeutici che possono modificare la prognosi (tabella 1).

\section{Overlap AMA M2 / SLA-LP}

Nei due casi SLA/LP positivi riscontrati nel nostro laboratorio, gli indici biochimici di epatite autoimmune di tipo I sono risultati nettamente prevalenti sugli indici di PBC. Per essi è stato quindi indispensabile il trattamento corticosteroideo in aggiunta all'acido urodesossicolico. 
Table I.

\begin{tabular}{|c|c|c|c|c|c|c|c|c|c|c|c|c|}
\hline $\begin{array}{l}\text { Parametri immunologici } \\
\text { associati }\end{array}$ & $\begin{array}{l}\text { AMA M2 } \\
\text { Totale } 40\end{array}$ & $\begin{array}{l}\text { Età } \\
\text { M:59 } \\
35-87\end{array}$ & FA & GOT & GPT & GGT & bil & $\lg G$ & $\lg M$ & $\begin{array}{l}\lg \\
\mathrm{A}\end{array}$ & Diagnosi & Terapia \\
\hline pANNA/ Spl00 inde $x>10$ & $1 / 40$ & 53aa & ++++ & ++++ & ++++ & +++ & $\begin{array}{l}++ \\
++\end{array}$ & + & $n$ & $\mathrm{n}$ & $\mathrm{AlH} / \mathrm{PSC}$ & $\begin{array}{l}\text { Cortisone } \\
\text { coleretico }\end{array}$ \\
\hline Anti SLA/LP & $2 / 40$ & $\begin{array}{l}59 \text { aa } \\
68 \mathrm{aa}\end{array}$ & ++ & ++ & ++ & ++ & ++ & ++++ & $\mathrm{n}$ & ++ & $\mathrm{AlH} / \mathrm{PBC}$ & $\begin{array}{l}\text { Cortisone } \\
\text { coleretico }\end{array}$ \\
\hline Gp2I0 (IFI,ELISA) & $6 / 40$ & $\begin{array}{l}68 \mathrm{aa} \\
54-83\end{array}$ & + & + & + & + & $-1+$ & $+1-$ & $+1-$ & $\mathrm{n}$ & $\begin{array}{l}\text { PBC/lieve } \\
\text { epatite } \\
\text { cronica }\end{array}$ & coleretico \\
\hline Spl00 Index $>10$ & $1 / 40$ & 39 aa & ++ & ++ & ++ & +++ & $\mathbf{n}$ & +++ & +++ & ++ & $\mathrm{AlH} / \mathrm{PBC}$ & $\begin{array}{l}\text { Cortisone } \\
\text { coleretico }\end{array}$ \\
\hline SpI00 (IFI,BLOT) Index $<10$ & $\begin{array}{l}5 / 40 \\
12,5 \%\end{array}$ & $\begin{array}{l}48 \mathrm{aa} \\
35-62\end{array}$ & $\mathbf{n}$ & + & + & + & $\mathrm{n}$ & $\mathrm{n}$ & $\mathrm{n}$ & $\mathrm{n}$ & $\begin{array}{l}\text { PBC/lieve } \\
\text { epatite } \\
\text { cronica }\end{array}$ & coleretico \\
\hline Pattern speckled $\bullet 1 / 320$ & $7 / 40$ & $\begin{array}{l}58 \mathrm{aa} \\
38-87\end{array}$ & ++ & ++ & ++ & ++ & $+1-$ & $\mathrm{n}$ & $+1-$ & $\mathrm{n}$ & $\begin{array}{l}\mathrm{PBC} / \mathrm{AlH} \text { a } \\
\text { lenta } \\
\text { evoluzione }\end{array}$ & $\begin{array}{l}\text { Coleretico } \\
\text { eventuale } \\
\text { Cortisone }\end{array}$ \\
\hline Anticentromero (IFI,blott) & $2 / 40$ & 55 aa & ++ & + & + & + & $+1-$ & + & $\mathrm{n}$ & + & $\begin{array}{l}\text { PBC/lieve } \\
\text { epatite } \\
\text { cronica }\end{array}$ & coleretico \\
\hline Anticentromero/SSARo52 & $1 / 40$ & 51 aa & ++++ & ++ & ++ & ++ & ++ & $\mathrm{n}$ & ++ & + & AlH/PBC & $\begin{array}{l}\text { Cortisone } \\
\text { coleretico }\end{array}$ \\
\hline SSA Ro 52 & $4 / 40$ & $\begin{array}{l}65 \mathrm{aa} \\
55-74\end{array}$ & ++ & ++ & ++ & ++ & $\mathrm{n}$ & ++ & ++ & $\mathrm{n}$ & $\mathrm{AlH} / \mathrm{PBC}$ & $\begin{array}{l}\text { Cortisone } \\
\text { Coleretico }\end{array}$ \\
\hline SSARo52/SSARo60 & $1 / 40$ & $56 a a$ & n & $-1+$ & $-1+$ & + & $\mathrm{n}$ & ++ & n & + & $\mathrm{PBC} / \mathrm{AlH}$ & Coleretico \\
\hline SSARo 60 & $1 / 40$ & 39 aa & $\mathbf{n}$ & $\mathrm{n}$ & $\mathrm{n}$ & $\mathrm{n}$ & $\mathrm{n}$ & $\mathrm{n}$ & $\mathrm{n}$ & $\mathrm{n}$ & asintomatica & $\begin{array}{l}\text { No terapia } \\
\text { specifica }\end{array}$ \\
\hline SSARo60/RNP-A/SSBLa & $1 / 40$ & $46 a a$ & n & $n$ & $n$ & $n$ & $\mathrm{n}$ & + & $n$ & + & $\begin{array}{l}\text { HCV trattato } \\
\text { Con INF }\end{array}$ & $\begin{array}{l}\text { Sospensione } \\
\text { INF }\end{array}$ \\
\hline Anti nucleosomi/MPO & $1 / 40$ & $60 \mathrm{aa}$ & + & $\mathrm{n}$ & + & ++ & $n$ & $\mathrm{n}$ & $n$ & $\mathrm{n}$ & $\begin{array}{l}\text { Reazione da } \\
\text { Isoniazide } \\
\text { vasculite }\end{array}$ & $\begin{array}{l}\text { Sospensione } \\
\text { terapia } \\
\text { specifica }\end{array}$ \\
\hline Anti nuclear lamins/MPO & $1 / 40$ & 65 aa & $n$ & $\mathrm{n}$ & $\mathrm{n}$ & $\mathrm{n}$ & $n$ & + & $\mathrm{n}$ & $\mathrm{n}$ & $\begin{array}{l}\text { Vasculite } \\
\text { sistemica }\end{array}$ & $\begin{array}{l}\text { Cortisone } \\
\text { ciclofosfamide }\end{array}$ \\
\hline $\begin{array}{l}\text { Assenza di overlap } \\
\text { immunologico }\end{array}$ & $\begin{array}{l}6 / 40 \\
15 \%\end{array}$ & $\begin{array}{l}58 \text { aa } \\
45- \\
79 \\
\end{array}$ & + & $\mathbf{n}$ & $\mathbf{n}$ & $+1-$ & $\mathbf{n}$ & $\mathbf{n}$ & $\mathbf{n}$ & $\mathbf{n}$ & $\begin{array}{l}\text { PBC } \\
\text { Biopsiat }\end{array}$ & coleretico \\
\hline
\end{tabular}

Overlap immunologico nei casi allo studio - tabella riassuntiva

Legenda: $n=$ nel range di normalità

//= non noto

$+/=$ debolmente positivo

$+=$ vari gradi di positività

Nelle statistiche anglosassoni (11) gli autoanticorpi anti SLA/LP sono riportati in circa il $30 \%$ dei pazienti con epatite autoimmune di cui alcuni con ANA, ASMA o anti LKM1 negativi. Essi tuttavia non configurano un quadro clinico e biochimico diverso da quello della AIH di tipo I per cui non permettono di identificare un terzo sottogruppo clinicamente distinto come Manns e collaboratori avevano proposto.

La ricerca di questi autoanticorpi è potenzialmente importante soprattutto per le implicazioni terapeutiche. In effetti i casi di cirrosi biliare primitiva che hanno caratteristiche di epatite autoimmune, sono rari ma rappresentano il maggior problema nella diagnosi differenziale delle epatiti autoimmuni rispetto ad altri disordini epatici: se la $\mathrm{PBC}$ ha una evoluzione lentissima, nel caso di overlap AIH/PBC un mancato trattamento corticosteroideo adeguato è associato ad una significativa morbilità e mortalità.

In uno studio tedesco (7) l'overlap PBC/SLA-LP era presente nel $3.9 \%$ della popolazione allo studio costituita da 233 casi di PBC in cui venivano evidenziate transaminasi e gammaglobuline significativamente più alte rispetto ai casi di $\mathrm{PBC}$ SLA-LP negative. Questi pazienti avevano un HLA caratteristico delle AIH e solo la terapia immunosoppressiva riduceva significativamente l'attività infiammatoria e la colestasi.

L'antigene SLA/LP non è specifico del fegato ed è una proteina citosolica. Esso non è chiaramente rilevabile in fluorescenza e va evidenziato con metodi ELISA o immunoblot.

Il significato degli autoanticorpi anti SLA/LP sembra essere stabilito da numerosi studi (9):

1) Sono markers specifici di epatite autoimmune

2) Nelle statistiche internazionali sono presenti approssimativamente nel $10-30 \%$ dei pazienti con epatiti autoimmuni con o senza autoanticorpi AMA o ASMA.

3) Aiutano ad identificare una porzione di pazienti con epatiti criptogenetiche che possono in realtà soffrire di epatiti autoimmuni.Questo ha conseguenze terapeutiche.

\section{AMA M2/ gp210}

La gp 210 è uno dei principali componenti, con la proteina $\mathrm{p} 62$, dell'antigene definito come nuclear 
pore complex ed è specificamente associata con pattern IFI perinucleare $(5,6)$. In uno studio di Invernizzi su di un ampio gruppo di soggetti si ammette che il rilievo di specifici autoanticorpi contro proteine nucleari dia rilevanti informazioni cliniche e che la ricerca di questi autoanticorpi possa aiutare nella diagnosi di $\mathrm{PBC}$, specialmente nei pazienti con bassi livelli di AMA. In questo ed in altri studi è stata fatta inoltre una stretta associazione tra un andamento più severo della $\mathrm{PBC}$ e la presenza di autoanticorpi anti nuclear pore complex. In particolare è stato confermato che:

1) la reattività anti nuclear pore complex è maggiore nei pazienti con $\mathrm{PBC}$ che nei controlli.

2) I pazienti PBC anti-NPC positivi presentano una malattia più severa indicata dalla evoluzione della cirrosi e delle sue complicanze.

3) Si ha inoltre associazione tra questi patterns ed un più marcato quadro infiammatorio: questi pazienti mostrano alterazioni dei test di funzionalità epatica e delle caratteristiche istologiche di infiammazione e danno epatocellulare.

4) La associazione tra positività anti-NPC e l'attività e la severità della malattia può essere di interesse pratico perché può contribuire ad identificare i pazienti che richiedono uno stretto follow up e un trattamento più aggressivo, come la somministrazione di steroidi.

5) La presenza di questo marker può far prospettare un overlap immunologico AIH/PBC (1).

Secondo l'esperienza da noi riportata, su quaranta sieri AMA M2 positivi, sei (15\%) erano contemporaneamente reattivi per $\mathrm{Ab}$ anti gp210. Essi presentavano indici ematochimici di sofferenza epatica più marcati rispetto ai sieri negativi per gli stessi autoanticorpi ma non tali da prospettare overlap AIH/PBC. Questi pazienti erano curati ambulatorialmente con acido urodesossicolico, ma con più stretto fallow-up rispetto ai pazienti affetti da PBC senza questo tipo di overlap.

\section{Overlap AMA M2/Sp100}

Ricerche sistematiche hanno recentemente dimostrato una associazione tra questa famiglia di autoanticorpi e un decorso clinico sfavorevole (4, 12). Studi incrociati su una ampia serie di sieri di pazienti con PBC ha correlato questi autoanticorpi con più marcate attività e severità della malattia; questo può essere di interesse pratico in quanto contribuisce a identificare pazienti che richiedono un trattamento più aggressivo; come la somministrazione di corticosteroidi.

Nella nostra casistica circa il $15 \%$ dei soggetti AMA M2 positivi con aspetti biochimici di PBC erano positivi anche per anti-Sp100, valutato sia in IFI che in ELISA. Due di essi presentavano aspetti di grave infiammazione epatica con dia- gnosi di AIH/PBC. Ambedue avevano livelli di $\mathrm{Ab}$ anti-Sp100 molto elevati, con index ELISA $>10$ prospettando una possibile incidenza del dato quantitativo sulla entità della malattia. Per essi la diagnosi era di overlap $\mathrm{AIH} / \mathrm{PBC}$ con necessità di trattamento steroideo.

I pazienti positivi per anti-Sp100 con index ELISA inferiore a 10 manifestavano invece, dal punto di vista clinico e biochimico, una lieve sofferenza dell'epatocita. Istologicamente era descritta una lieve epatite cronica da inquadrare nel contesto clinico. Nessuno di essi era in trattamento steroideo (figura I).

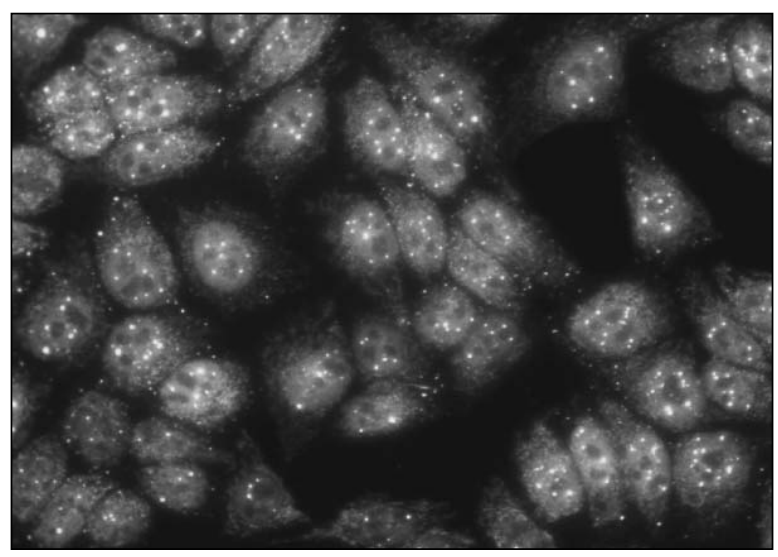

Figura I. Pattern nuclear dots (Sp I00)/AMA su cellule HP2

\section{AMA M2/ SSARo52}

L'associazione con SSARo52 è frequente in pazienti AMA M2 positivi e può essere di difficile definizione in fluorescenza per la localizzazione citoplasmatica dell'antigene SSARo52. I sei pazienti positivi per questi autoanticorpi, tra $\mathrm{i}$ quaranta allo studio (complessivamente il 12\%), presentavano un andamento severo della PBC con marcati indici di sofferenza epatica. Per essi si prospettava uno stretto fallow-up per il sospetto di overlap AIH/PBC.

In due pazienti c'era inoltre una doppia positività auto $\mathrm{Ab}$; in particolare un paziente con contemporanea positività per anticorpi anti centromero aveva prognosi peggiore e configurava un franco overlap $\mathrm{AIH} / \mathrm{PBC}$ con necessità di trattamento corticosteroideo; nell'altro caso la presenza di SSARo60 non sembra essere un ulteriore fattore aggravante.

Per capire il possibile ruolo degli anti SSARo52 nelle epatopatie è interessante la pubblicazione di Liaskos et al. (8) che ha studiato la cross reattività tra autoanticorpi anti SLA/LP e anticorpi antiSSARo52 in pazienti positivi per ambedue le specificità. In particolare il $98 \%$ dei sieri di pazienti SLA/LP positivi reagivano con SSARo52 ed i due tipi di autoanticorpi avevano simile comportamento ed identico profilo delle 
sottoclassi IgG; gli autoanticorpi verso le due specificità non si inibivano a vicenda. Secondo gli autori i pazienti reattivi verso questi due antigeni hanno una malattia più severa sia dal punto di vista biochimico che da quello istologico. La sieropositività verso i due tipi di autoanticorpi non è dovuta a cross reattività ed ha significato prognostico in quanto è un marker di AIH di maggiore gravità se comparato con $\mathrm{i}$ casi negativi.

Il fatto che autoanticorpi anti SSARo52 siano peggiorativi per la funzionalità epatica è confermato dallo studio di Muratori et al. (3) secondo il quale il 28\% dei pazienti con PBC reagiva contro l'ENA SSARo52. Questi pazienti avevano, al momento della diagnosi, uno stadio istologico più avanzato ed un più alto livelli sierico di bilirubina e di IgM rispetto ai negativi. Per questi autori quindi gli autonticorpi anti SSARo52 e anti centromero hanno una alta specificità per la $\mathrm{PBC} \mathrm{e}$ possono essere così di rilevanza diagnostica nei casi negativi per AMA. Inoltre gli anti SSARo52 possono identificare pazienti con una $\mathrm{PBC}$ più attiva e avanzata.

\section{M2/ anticentromero}

La presenza di anticorpi anticentromero associati ad AMA M2, in due casi allo studio, aggiungeva alla PBC gli aspetti biochimici e istologici di una lieve epatite cronica. Non erano riportati, al momento dell'osservazione, segni di sclerodermia segnalati in una minoranza di pazienti affetti da PBC. Sia la sclerodermia che la PBC sono caratterizzate istologicamente da un basso grado di infiammazione e fibrosi cronica dei rispettivi organi bersaglio.

In uno studio di McHugh (10) non è stata evidenziata cross reattività tra AMA e ab anti polipeptidi centromerici; la loro associazione peggiora comunque la prognosi e può richiedere l'uso di corticosteroidi. Un esempi di associazione AMA M2/anticentromero in IFI è riportato nella figura II.

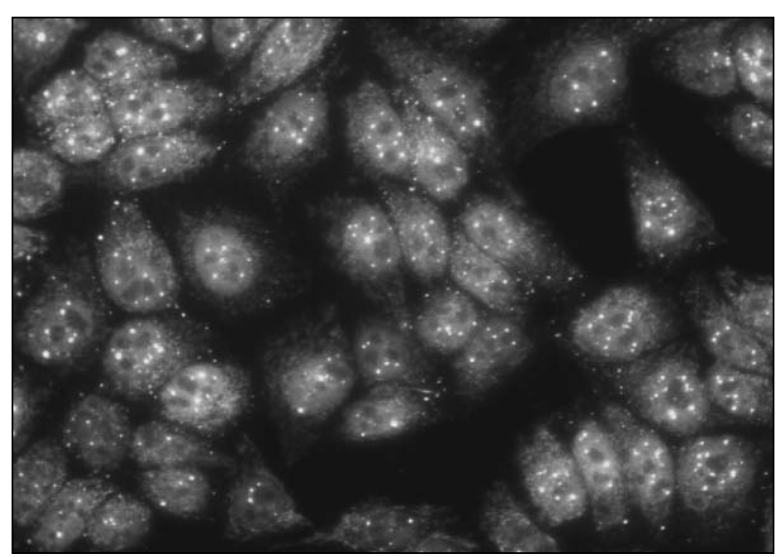

Figura II. overlap AMA M2/anticentromero in IFI
M2/positività speckled in IFI $\geq 320$.

Questa positività ANA è piuttosto frequente e, tipicamente non si associa ad alcuna specificità ENA (da noi testata anche in Western Blot). Essa può essere valutata solo in fluorescenza e non va trascurata in quanto, anche in base alla nostra esperienza, complica l'andamento classico della PBC con chiari segni di sofferenza dell'epatocita. Gli indici biochimici di sofferenza epatica persistono a bassi livelli per molto tempo e inizialmente non richiedono trattamento steroideo. La loro presenza è comunque un motivo di aggravamento della sintomatologia; lo stimolo immunologico aggiuntivo continuo richiederà ad un certo punto del corso naturale della malattia l'uso di immunosoppressori.

I sette pazienti positivi (17\%) per questo tipo di overlap valutati nella nostra casistica presentavano nettamente segni di sofferenza dell'epatocita; solo quelli in fase più avanzata assumevano corticosteroidi. In tutti la biopsia evidenziava AIH.

I due possibili aspetti in IFI, AMA M2 e overlap AMA M2/ANA, sono riportati nelle figure III e IV.

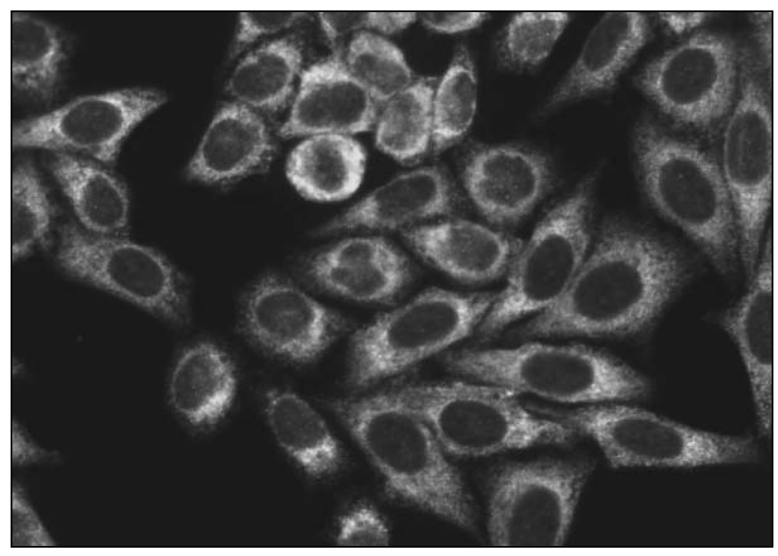

Figura III. AMA su vetrini HP2

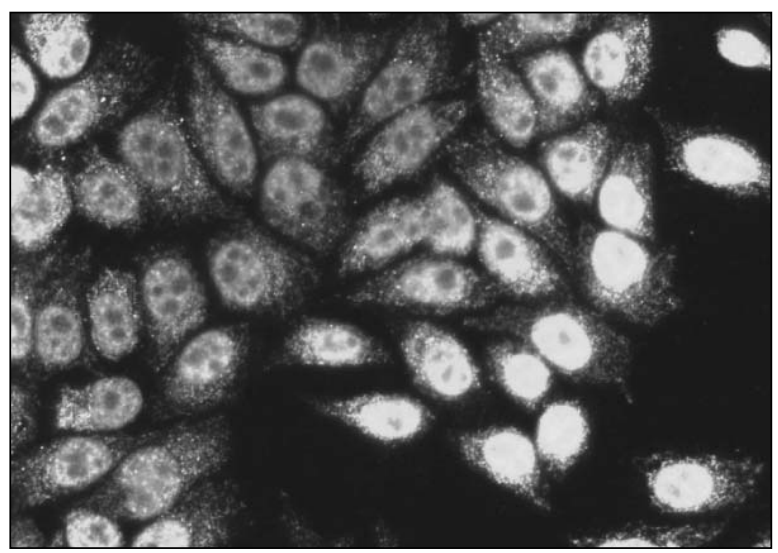

Figura IV. Overlap AMAIANA speckled su vetrini HP2 
Overlap AMA M2

con SSARo60 e/o SSB, nRNP, ANCA

L'associazione con ENA diversi da SSARo52 non sembrano peggiorare la prognosi a livello epatico in pazienti AMA M2 positivi. Alcuni di questi casi erano asintomatici e venivano monitorati nel tempo in quanto si prospettava la possibile insorgenza di manifestazioni immunologiche sistemiche (2). Allo stato attuale delle conoscenze non è noto quale sia il movente che induce il passaggio da alterazione immunologica a malattia immunologica. In due casi era presente un overlap AMA M2 /MPO con sintomi di vasculite sistemica senza però segni specifici di sofferenza epatica.

\section{Un caso particolare}

AMA M2/pANNA/sp100(index>10)

La forma clinicamente più grave si è avuta con un con un quadro sierologico caratterizzato dalla associazione di tre tipi di autoanticorpi contro antigeni sia delle vie biliari che dell'epatocita: AMA M2, Sp100 (index>10) e pANNA. In base ai risultati sierologici si dovrebbe trattare di una aggressione diretta contro tutte le componenti epatiche, con netta prevalenza dell'overlap AIH/PSC e con l'aggravante di una positività AMA M2.

Clinicamente si è assistito ad una evoluzione tanto rapida e grave da prospettare la necessità di trapianto in una donna di 53aa $(\mathrm{GOT}=1314$, $\mathrm{GPT}=935$, gamma $\mathrm{GT}=389$, bilirubina $=24, \mathrm{FA}$ $=1174$ ). La sintomatologia ed i dati ematochimici sono progressivamente migliorati con terapia steroidea. Nella figura $\mathrm{V}$ è riportato l'aspetto pANNA in IFI del caso descritto.

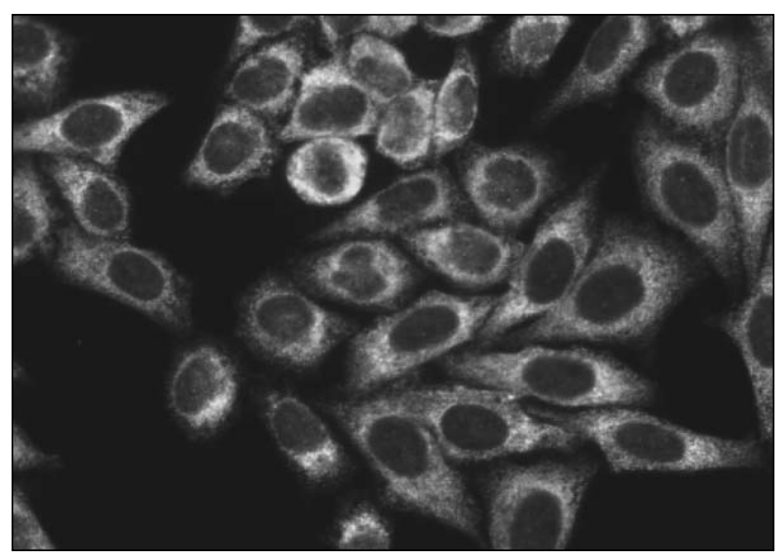

Figura V. Aspetto pANNA in IFI su vetrini fissati in etanolo. Fluorescenza negativa su vetrini fissati in formalina

\section{DISCUSSIONE}

La coesistenza di autoanticorpi AMA M2 con altre specificità autoanticorpali è molto frequente (85\% dei casi allo studio) anche in una casistica limitata come può essere quella di un'esperienza locale. Il 15\% dei pazienti che presenta solo posi- tività AMA M2 ha una malattia lentamente evolutiva ben controllata dal solo trattamento con acido urodesossicolico e compatibile con una normale durata della vita. Abbiamo osservato che il complicarsi del quadro immunologico modifica l'andamento della malattia in vario modo, influendo sulla sua gravità e sulla rapidità evolutiva. Conoscere l'assetto immunologico e il suo significato comporta l'impostazione un corretto follow up e di una corretta prognosi; è possibile inoltre discriminare tra casi che necessitano o necessiteranno di trattamento immunosoppressore.

Le associazioni più comuni sono quelle che comportano un peggioramento della funzionalità epatica in quanto configurano un overlap AIH/PBC. I quadri infiammatori più gravi si hanno per positività $\mathrm{SLA} / \mathrm{LP}$ e per alti livelli di $\mathrm{sp} 100$ (index $>10$ ) in cui prevale la diagnosi di AIH di tipo $\mathrm{I}^{\circ}$. Seguono gli autoanticorpi SSARo52 e gli anticentromero che, specie se presenti contemporaneamente, danno un franco overlap AIH/PBC.

In un'alta percentuale di casi $(17 \%)$, abbiamo osservato l'associazione con positività ANA $>1: 320$, con pattern esclusivamente speckled ed ENA negativo. Questo aspetto va sempre valutato con attenzione perché può richiedere, a lungo termine, un trattamento corticosteroideo in quanto overlap AIH/PBC a lenta evoluzione.

In più del $30 \%$ dei casi complessivamente si ha poi associazione con autoanticorpi anti-gp210 o bassi livelli di Ab anti-Sp100, secondo la letteratura marker sicuri di flogosi epatica. Nella nostra casistica questo tipo di overlap aggiunge alla PBC gli aspetti di una epatite cronica non ben delineata dal punto di vista biochimico e istologico. Il coinvolgimento dell'epatocita modifica comunque la prognosi e la prospettiva terapeutica.

Poco frequenti sono altre associazioni che nel nostro caso riguardavano anti MPO e alcuni ENA presenti in connettiviti (SSARo60, RNP-A, SmD, SSB-LA). Essi non peggiorano la funzionalità epatica e in questi casi la qualità di vita, la prognosi e la terapia dipendono dall'innescarsi di malattie autoimmuni sistemiche.

\section{BIBLIOGRAFIA}

1. Chazouilleres O, Wendum D, et al. Primary biliary cirrhosis-autoimmune hepatitis overlap syndrome: clinical features and response to therapy; Hepatology 1998; 28: 296-301.

2. Csepregi A, Szdoray P, Zeher M, Do autoantibodies predict autoimmune liver disease in primary Sjogren's sindrome? Data of 180 patients upon a 5 year followup; Scand J Immunol 2002 Dec; 56(6): 623-9.

3. Granito A, Muratori P, et al. Antibodies to SS-A/Ro52kD and centromere in autoimmune liver disease; a clue to diagnosis and prognosis of primary biliary cirrhosis. 
Aliment Pharmacol Ther 2007 sep 15; 26(6): 831-8.

4. Guldner HH, Szostecki C, et al. IFN enhance expression of Sp100, an autoantigen in primary biliary cirrhosis. J Immunol 1992 Dec 15; 149(12): 4067-73.

5. Invernizzi $\mathrm{P}$, Podda $\mathrm{P}$, et al. Autoantibodies against nuclear pore complexes are associated with more active and severe liver disease in primary biliary cirrhosis. Journal of Epatology 2001; 34: 366-72.

6. Itoh S, Ichida $\mathrm{T}$, et al. Autoantibodies against a 210 $\mathrm{kDa}$ glycoprotein of the nuclear pore complex as a prognostc marker in patients with primary biliary cirrhosis. J Gastroenterol Hepatol 1998; 13: 257-65.

7. Kanzler S, Bozkurt S, et al. Presence of SLA/LP autoantibodies in patients with primary biliary cirrhosis as a marker for secondary autoimmune hepatitis (overlap syndrome). Dtsch Med 2001 Apr 20; 126(16): 450-6.

8. Liaskos C, Bogdanos DP, et al. Antibody responses specific for soluble liver antigen co-occur with Ro52 autoantibodies in patients with autoimmune hepatitis. Poster on SLA/Ro52 at EASL 2007 Barcelona. J Hepatology 2007; 1 (suppl, 1): 250.

9. Manns MP. Antibodies to soluble liver antigen: specific marker autoimmune hepatitis. J Hepatol 2000 Aug; 33(2): 208-15.

10. McHugh NJ, James IE, et al. Autoantibodies to mitochondrial and centromeres antigens in primary biliary cirrhosis and systemic sclerosis. Clin Exp Immunol 1990; 81: 244-9.

11. Wies I, Brunner S, et al. Identification of target antigen for SLA/LP autoantibodies in autoimmune hepatitis. Lancet 2000 Apr 29; 355(9214): 1510-5.

12. Zuchner D, Sternsdorf T, et al. Prevalence, kinetics, and therapeutic modulation of autoantibodies against $\mathrm{Sp} 100$ and promyelocytic leukaemia protein in a large cohort of patients with primary biliary cirrhosis. Hepatology 1997; 26: 1123-30. 